\title{
Che cosa riguarda la critica dei farisei in Mt 23,25-26?
}

Nel Nuovo Testamento possiamo osservare diversi gruppi di persone che si oppongono a Gesù e al Suo insegnamento: i farisei, i sadducei, i capi dei sacerdoti e gli scribi. Questi gruppi sono differenti fra di loro, hanno diversi scopi da realizzare e diversi motivi per opporsi a Cristo. Nei Vangeli, vengono spesso considerati in modo molto negativo.

Secondo l'opinione tradizionale, i farisei nei Vangeli sinottici sono considerati come i nemici di Gesù e dei cristiani. La loro presentazione è tendenziale e unilaterale, limitata a questioni controverse ${ }^{2}$. I farisei, come si osserva in diversi testi, furono introdotti nel Nuovo Testamento come il simbolo degli avversari di Gesù. Gli Evangelisti omettono quello che è, nella vita dei farisei, positivo e concorde con i cristiani ${ }^{3}$. La loro presentazione è più complicata ma questa non è l'oggetto di questo studio.

Nel presente lavoro vorrei concentrarmi sulla situazione che Matteo descrive in 23,25-26. Lo scopo è presentare Gesù che non critica soltanto i farisei che rispettano le regole della purezza rituale nella vita quotidiana, ma che anzitutto fa vedere che cosa è peccato nell'atteggiamento dell'uomo, sia nella vita di un fariseo, che nella vita di ognuno. Questo lavoro mostra che durante la discussione di Gesù con i farisei sul fenomeno dell'ipocrisia, Egli, il Maestro, non vuole rifor-

1 Tomasz Krzysztofiak - licenziato presso il Pontificio Istituto Biblico nel 2016, ha conseguito il Dottorato in Teologia nel 2020. È docente presso il Primaziale Seminario Maggiore di Gniezno e presso l'Università Adam Mickiewicz di Poznań, e-mail: tomkrzy2@gmail.com, ORCID: 00000002-2347-1249.

2 Cfr. W. Rakocy, Obraz i funkcja faryzeuszy w dziele Eukaszowym ( $₫ k-D z)$. Studium literacko-teologiczne, Lublin 2000, p. 13.

3 Cfr. W. Rakocy, Faryzeusze. Historia - Ewangelie, Lublin 2002, p. 167. 
mare la legge rituale, ma cambiare il comportamento dell'uomo, tramite il pentimento e il rinnovamento della vita.

\section{I farisei}

Descrivere i farisei dal punto di vista storico è molto difficile a causa della natura delle fonti e della loro contraddizione. Le fonti principali che trattano dei farisei sono: i lavori scritti di Giuseppe Flavio, gli apocrifi, la letteratura rabbinica, i testi di Qumran, il Nuovo Testamento. Nonostante non siano troppo numerosi, tutti i testi collocano l'attività dei farisei nella terra di Israele, nel periodo che va dalla dinastia degli Asmonei fino alla distruzione del tempio di Gerusalemme.

Le prime informazioni sui farisei si trovano nel II sec. a.C., durante la rivolta dei Maccabei, quando, nel 166 a.C., i Maccabei lottarono contro Antiochus IV Epiphanes che governava nei territori della Siria e della Palestina ${ }^{4}$. La lingua greca descrive questo gruppo come $\Phi \alpha \rho \iota \sigma \alpha i ̂ \varsigma$ ("pharisaîos"). Non si sa con certezza né che cosa significhi questa parola, né se essa sia il nome che i farisei si erano scelti. La rarità dell'uso nelle fonti antiche la parola "pharisaîos" ed anche l'uso decisamente polemico o spregiativo del termine rendono abbastanza probabile la tesi che non è stato un nome scelto originariamente dai membri stessi del gruppo 5 . È probabile che il termine significhi "qualcuno che è separato", "è separatista", "separato" (da peccato, impurità); indica quella parte di uomini che, in modo particolare, osservava la legge ${ }^{6}$. I farisei, infatti, vengono spesso considerati come una confraternita non sacerdotale di eruditi, esperti nell'interpretazione delle Scritture; essi si consideravano pii e devoti nelle pratiche religiose ${ }^{7}$.

La parola "pharisaîos" si basa sulle lingue semitiche e viene dal termine "perushim". La radice nella lingua ebraica crea un verbo פרש (prš) che significa "essere disperso", "qualcuno che è separato" .

Nonostante nella lingua contemporanea l'immagine dei farisei venga usata come sinonimo di ipocriti e di formalista, tuttavia, non possiamo dimenticare la connotazione positiva: i farisei sono gli uomini che si sono separati per condurre la vita che piace a $\mathrm{Dio}^{9}$. In genere, $\mathrm{i}$ farisei sembrano individui di un ceto ele-

4 Cfr. K. Kern, We are the Pharisees, Scottdale 1995, p. 31.

5 Cfr. J. Sievers, Chi era un fariseo? Un approccio prosopografico, "Poznańskie Studia Teologiczne" 27(2013), p. 14.

6 Cfr. $\Phi \alpha \rho \iota \sigma \alpha i o \varsigma$, in: A Greek-English lexicon of the New Testament and other early Christian literature, ed. W. Bauer, F. W. Danker, W. F. Arndt, F. W. Gingrich, Chicago ${ }^{32000, ~ p . ~ 1049 ; ~}$ U. Luz, Vangelo di Matteo, Vol. 3, Brescia 2013, p. 446.

7 Cfr. U. Luz, op. cit., p. 446.

8 Cfr. $\varpi \rho \pi$, in: P. Reymond, Dizionario di ebraico e aramaico biblico, Roma ${ }^{22} 212$, p. 343.

9 Cfr. E. Dąbrowski, Nowy Testament na tle epoki, t. 1, Poznań ${ }^{2} 1965$, p. 183. 
vato, ma non hanno potere autonomo o indipendente. Forse il termine più adatto rimane "scuola di pensiero" 10 .

Con alcune descrizioni storiche, possiamo oggi ricostruire un quadro relativo ai farisei.

\section{Giuseppe Flavio}

Giuseppe Flavio, storico ebreo, parla dei farisei in "Guerra giudaica", "Antichità", e "Autobiografia". Il suo lavoro si riferisce al mondo pagano, soprattutto ai Romani. Per questo motivo, egli presenta le tre principali fazioni religiose, i farisei, i sadducei e gli esseni, come scuole filosofiche e descrive le loro idee. Giuseppe Flavio condanna tutto ciò che porta le tracce della ribellione e della rivolta contro Roma ed elogia l'approccio pacifico. Egli pone le origini dei farisei nel tempo di Jonathan, il successore di Giuda Maccabeo e la loro scomparsa nei primi due secoli d.C. ${ }^{11}$. Secondo l'autore, più di 6000 farisei rifiutarono di prestare la loro fedeltà ad Erode. Ci informa anche di un gruppo di Ebrei che sono rimasti fedeli alle tradizioni dei loro antenati e hanno rispettato le leggi che Dio ha dato a Mosè sul monte Sinai. Egli descrive quel gruppo come una forte comunità, con una grande influenza sulla vita politica ${ }^{12}$. Sottolinea anche i diversi luoghi in cui i farisei si sono distinti come esperti della legge.

\section{Qumran}

Le scoperte del Mar Morto fanno riferimento ai farisei, ma nemmeno una volta li menzionano per nome. Le scritture di Qumran si concentrano sulla critica dei farisei circa l'aspetto legale. Essi, probabilmente, potevano essere in conflitto con la comunità di Qumran a causa delle vicende politiche durante il regno di Alexander Jannaeus. Secondo la comunità di Qumran, erano colpevoli della rottura dell'alleanza, dell'allontanamento dalla Torah e dalla giustizia e anche dell'interpretazione impropria della Torah ${ }^{13}$.

${ }^{10}$ Cfr. J. Sievers, op. cit., p. 25.

${ }^{11}$ Cfr. Dictionary of Jesus and the Gospels, ed. J. B. Green, J. K. Brown, N. Perrin, Downers Grove 1992, pp. 608-610.

12 Josephus, Jewish Antiquities, Vol. VII: Books 16-17, trans. R. Marcus, A. Wikgren. Loeb Classical Library 410. Cambridge (MA), 1963, pp. 391-393.

13 Cfr. W. S. Green, What do we really know about the Pharisees, and how do we know it?, in: Quest of the Historical Pharisees, ed. J. Neusner, B. D. Chilton, Waco 2007, p. 411. 


\section{La letteratura rabbinica}

Le informazioni sui farisei provengono anche dalla letteratura rabbinica - nella Mishnah (include le norme che provengono dall'interpretazione della Torah), nel Talmud (i commenti e le discussioni che vengono dall'interpretazione della Mishnah) e nel Midrash (l'interpretazione della Torah, versetto per versetto). Queste opere sono più tardive e contengono informazioni molto diverse. Purtroppo, non sono molto utili per conoscere la situazione dei farisei prima dell'anno 70 d.C. ${ }^{14}$.

\section{Gli apocrifi}

Gli apocrifi non menzionano esplicitamente i farisei. Informano soltanto sulla percezione dei farisei nei secoli successivi e non forniscono tante informazioni storiche. Le informazioni contenute negli apocrifi spesso distorcono la realtà ${ }^{15}$. Il valore positivo degli apocrifi emerge dalla loro provenienza dall'ambiente dei farisei (PsSal), oppure dalle fonti che contengono le loro opinioni (2 Bar, 4 Ezra). Tuttavia, è anche possibile che gli apocrifi vengano dall'ambiente vicino ai farisei, ma non riflettano strettamente le loro opinioni ${ }^{16}$.

\section{Il Nuovo Testamento}

Nel Nuovo Testamento, i farisei sono stati coinvolti nella interpretazione della legge: la polemica di Gesù con loro ha un carattere religioso - legale. La parola "fariseo" ha spesso connotazioni negative. I Vangeli presentano i farisei come avversari e nemici di Gesù. Questi critica spesso il loro atteggiamento e la loro ipocrisia (Mt 23,28; Mc 12,15; Lc 12,1) e ciò, poi viene sviluppato anche nella tradizione cristiana. Ciononostante, nel Nuovo Testamento si trovano anche immagini positive dei farisei, per es.: il fariseo Nicodemo che è venuto da Gesù durante la notte ( $\mathrm{J} 3,1-21)$, difende Gesù dai nemici $(\mathrm{J} 7,50-53)$ ed è presente alla sua sepoltura (J 19,39) ${ }^{17}$. Il Vangelo non condanna l'individuo, i farisei conosciuti in sé, ma un atteggiamento generale, cioè l'ipocrisia di diversi farisei ${ }^{18}$.

Le informazioni colte da queste fonti non sono esplicite ed univoche. Contengono diverse considerazioni sulla vita quotidiana dei farisei, sul loro numero

14 Cfr. W. Rakocy, Faryzeusze..., op. cit., pp. 27-29.

15 Cfr. R. Rubinkiewicz, Wprowadzenie do apokryfów starego Testamentu, Lublin 1987, pp. $72-75$.

16 Cfr. W. Rakocy, Faryzeusze..., op. cit., pp. 25-26.

17 Cfr. P. C. Bosak, Leksykon wszystkich postaci biblijnych, Kraków 2015, pp. 482-483.

18 Cfr. P. Polak, Fundamentalizm faryzejski a radykalizm ewangeliczny, "Eryda" 1(1)2015, p. 49. 
o sulle loro regole di vita. I farisei, nei Vangeli di Matteo e Giovanni, sono descritti come avversari di Gesù, sono responsabili della sua morte (per es. Mt 12,14; 22,15; Gv 7,32; 11,47-53). Un'immagine più clemente si trova nel Vangelo di Marco e nel Vangelo di Luca, dove i farisei, da una parte, sono condannati, ma dall'altra non sono descritti come responsabili della crocifissione di Cristo. Si vede anche una certa intimità di Gesù (per es. Lc 7,36 o 11,37) con il fariseo che lo invita a tavola. Gli Atti degli Apostoli presentano Gamaliele, che era un insegnante saggio della legge, aperto alle diverse opinioni (At 5,38-39). Anche Paolo, in Fil 3,5, non nega le sue radici farisaiche. Si deve ricordare che il Nuovo Testamento non si concentra sulla presentazione dei farisei, ma sugli insegnamenti di Gesù ${ }^{19}$.

Il Nuovo Testamento presenta un'immagine spirituale dei farisei ed anche dei sadducei come persone che richiedono a Gesù un segno straordinario e, allo stesso tempo, non accettano tanti suoi miracoli. Essi sono presentati come avversari e oppositori dell'insegnamento del Maestro di Nazareth. Ma, è necessario vedere che $\mathrm{i}$ farisei e i sadducei erano in contrasto più tra loro che con Gesù stesso ${ }^{20}$.

I Vangeli si sono sviluppati sui materiali tradizionali che facevano parte della memoria dei discepoli di Gesù. Ogni Vangelo è unico, originale, scritto secondo la volontà e capacità di ciascuno degli Evangelisti. Grande parte del materiale dei Vangeli sinottici presenta l'immagine dei farisei in termini molto negativi. Tuttavia ci sono alcuni passi dove la presentazione di questo gruppo è migliore. Questi frammenti, probabilmente, furono scritti negli anni settanta del I secolo, perché, poi, dopo la caduta del Tempio di Gerusalemme, le relazioni fra i cristiani e i giudei sono cambiate ${ }^{21}$.

Bultmann prova a rispondere al motivo per cui i Vangeli presentano un'immagine negativa dei farisei. L'autore afferma che i dialoghi polemici fra Gesù e i farisei nei Vangeli non sono collegati direttamente con il ministero di Gesù, ma sono determinati dalle esigenze delle prime comunità cristiane. Secondo Bultmann, è molto probabile che queste polemiche rappresentino piuttosto i problemi e le polemiche interne delle prime comunità cristiane ${ }^{22}$. Anche H. Maccoby indica, per esempio, che il Nuovo Testamento contiene poche informazioni per quanto riguarda la purezza rituale. Secondo la sua opinione, non c'è nessun motivo per sostenere che il comportamento di Gesù, che siede a tavola con i peccatori e i pubblicani (Mt 9,10-12), si trovi in opposizione con i farisei e la purezza rituale. Gli esattori delle tasse venivano trattati come criminali e peccatori che collabora-

19 Cfr. A. J. Saldarini, Farisei, scribi e sadducei nella società palestinese, Brescia 2003, pp. 167-168.

20 Cfr. M. Rosik, Śladami faryzeuszy i saduceuszy, "Ziemia Święta” 2(2007)50, pp. 16-17.

21 Cfr. R. A. Wild, The encounter between Pharisaic and Christian Judaism: some early Gospel evidence, "Novum Testamentum" 27/2(1985), pp. 112-113.

22 Cfr. R. Bultmann, The History of the Synoptic Tradition, New York ${ }^{2} 1968$, pp. 48-49. 
vano con gli occupanti romani, raccogliendo le tasse per i nemici di Israele. Anche un frammento di Mishnah (M. Toh. 7:6) sottolinea che gli esattori delle tasse erano particolarmente impuri. Queste obiezioni contro gli esattori delle tasse non si riferivano dunque alla purezza rituale, ma ai loro contatti con gli occupanti ${ }^{23}$.

\section{I farisei e la purità rituale}

Uno degli elementi regolarmente presenti nell'intera evoluzione del pensiero teologico, dalle origini della Torah fino alla riflessione rabbinica dei primi secoli dell'era cristiana, è imparare a distinguere questo che è puro טהר (ṭh) da quello che è impuro טמא (țm'). La purità rituale ${ }^{24}$ è lo stato che permette all'uomo di contattare Dio. La mancanza della purità, cioè lo stato dell'impurità, porta una estromissione del contaminato da alcune circostanze della vita dell'uomo, dalla partecipazione agli atti rituali propri della società in cui l'individuo vive, fino all'esclusione totale dalla vita della comunità per un periodo meno o più lungo in base alla gravità della contaminazione ed alla durata delle purificazioni prescritte. L'impurità rituale viene, anzitutto, causata da un evento naturale, ad esempio, la nascita o la morte di una persona, i rapporti sessuali, il mangiare alcuni alimenti particolari, l'insorgere di alcune malattie ${ }^{25}$.

Secondo le prescrizioni date dalla Torah, solo i membri della casta sacerdotale erano tenuti all'abluzione rituale delle loro mani e dei piedi prima di compiere atti di culto, ad esempio, l'offerta d'incenso a Dio o il cibarsi delle parti delle vittime sacrificali a loro spettanti (Es 30,17-23; 40,30-32). In seguito, si era gradualmente attestato, anche presso alcuni gruppi, l'uso di lavarsi le mani prima di ogni pasto ${ }^{26}$.

23 Cfr. H. Maccoby, Ritual and morality. The Ritual Purity System and its Place in Judaism, Cambridge 1999, pp. 149-150.

24 Nel corso dei secoli, concezioni di purità e di impurità si sono evolute, hanno viaggiato parallelamente fino ad affiancarsi e, in alcuni casi, a sovrapporsi: si tratta di individuare la diversità e la somiglianza tra il concetto di "impurità rituale" e di "impurità morale". La seconda categoria, 1' "impurità morale", viene causata da una trasgressione di tipo etico. In Lev 18, questi atti vengono descritte come "cose abominevoli", oppure "nefandezze". Tra queste, l'autore biblico colloca l'idolatria, i peccati di natura sessuale o l'omicidio. L'impurità morale è sempre frutto di un grave peccato, per il quale non esistono purificazioni possibili, ma soltanto punizioni che vanno dall'allontanamento dal Tempio fino all'esilio; cfr. A. S. Romano, Purità ed impurità tra ebraismo e tradizioni sinottiche. Casi di impurità corporale, https://www.academia.edu/31802505/Purit\%C3\%A0_e impurit\%C3\%A0_tra_ebraismo_e_tradizioni_sinottiche_Casi_di_impurit\%C3\%A0_corporale [accesso 05.01.2021], p. 12.

25 Cfr. Ibidem, pp. 11-12.

26 Cfr. Ibidem, p. 185. 
Importante è osservare che la tradizione biblica e giudaica riteneva che la purità rituale ha un valore etico e che gli Israeliti sono più impuri quando non osservano la Legge piuttosto che quando mancano di purità rituale ${ }^{27}$.

La purezza rituale era importante nella vita quotidiana dei farisei. Essi mantenevano le loro tradizioni anche al di fuori del Tempio, nelle proprie case. Il cibo quotidiano doveva essere mangiato in stato di purezza rituale. In questo modo, i farisei cercavano di diventare simili ai sacerdoti del Tempio ${ }^{28}$. Questo riferimento ai sacerdoti era diventato per i farisei molto importante. Consideravano il cibo come santo e rifiutavano di mangiare tutto ciò che poteva essere ritualmente contaminato (per es. ciò che aveva avuto contatto con un cadavere) ${ }^{29}$. Un altro autore, Rivkin, sostiene che i farisei formavano un grande gruppo di persone che si concentravano sull'insegnamento della Legge. Secondo lui, non erano separatisti, ma si impegnavano pienamente nella vita politica e religiosa ${ }^{30}$.

Secondo Neusner, i farisei potevano mangiare soltanto con le persone giuste, cioè quelle che ubbidivano alla legge della purezza e ad altre abluzioni rituali. Tale tradizione deriva da una legge antica che richiedeva di lavare le mani prima di mangiare. Egli scrive che i farisei mangiavano solo i cibi adatti per non essere impuri, mangiavano con grande attenzione, con le mani pulite, avendo cura della purezza rituale dei piatti ${ }^{31}$.

\section{Gesù e i farisei in Matteo 23,25-26}

Il capitolo 23 del Vangelo secondo Matteo presenta un discorso da cui si evincono le relazioni negative in cui, da una parte, ci sono Gesù e i suoi discepoli e, dall'altra, gli scribi e i farisei. Mt 23,13-33, in cui si trova Mt 23,25-26, è una pericope costruita da sette invettive minacciose di varia lunghezza, introdotte tutte dal termine oúaı, (ouaí) "guai". L'arringa di Gesù comincia con il regolare intercalare della formula: "Guai a voi, scribi e farisei ipocriti", sconosciuta nel greco profano. Invettive simili ricorrono nella tradizione profetica (Is 5,8-24; 10,1-11; 28-33; Am 5,16-6,11). I "guai" rappresentano un atto comunicativo che può veicolare tre messaggi diversi: quello di lamentazione o paura; quello di

${ }_{27}$ Cfr. A. Paciorek, Ewangelia wedtug św. Mateusza. Rozdziaty 14-28. Wstęp, przektad z oryginatu, komentarz, t. 2, Częstochowa 2008, p. 415.

28 Cfr. J. Neusner, From Politics to Piety: The Emergence of Pharisaic Judaism, New York 1973, p. 83.

${ }_{29}$ Cfr. H. K. Harrington, Did the Pharisees eat ordinary food in a state of ritual purity, "Journal for the Study of Judaism" 26/1(1995), pp. 53-54.

30 Cfr. K. G. Newport, The Pharisees In Judaism prior to a. d. 70, “Andrews University Seminary Studies" 29/2(1991), pp. 128-129.

31 Cfr. J. Neusner, op. cit., pp. 79-80. 
invettiva nel senso di un severo rimprovero; quello di una minaccia, espressa con i verbi al futuro. Il testo di Matteo sembra essere più vicino al genere dell'invettiva. Gesù rimprovera perché vuole la conversione degli ascoltatori ${ }^{32}$; alla formulazione fa seguito la motivazione dell'esclamazione. I due versetti di Mt 23,25-26 creano la quinta invettiva.

In Mt 23,25, gli scribi e i farisei sono accusati perché puliscono l'esterno di piatti e bicchieri, mentre dentro sono riempiti con il frutto della rapina e dell'intemperanza. La distinzione fra la parte interna e quella esterna di un vaso è un dato ben noto a quanti erano a conoscenza dei precetti di purità degli scribi. L'invettiva si prende gioco della sottile distinzione tra parte esterna e interna di un recipiente, non criticando sarcasticamente una prassi reale dei farisei, ma minimizzando, usando una iperbole retorica, giungendo al risultato, chiaramente irreale, della divisione tra interno e esterno. Gli avversari sono quindi presentati in modo caricaturale 33 .

A colui che descrive l'invettiva non interessa la mera purità rituale. È interessante osservare che l'intervento compie una nuova sterzata inaspettata: "All'interno sono pieni di rapina e d'intemperanza". La parola $\dot{\alpha} \rho \pi \alpha \gamma \eta$ (harpagē) indica la "rapina" o "quanto è stato rapinato", raramente in senso soggettivo "l'avidità";

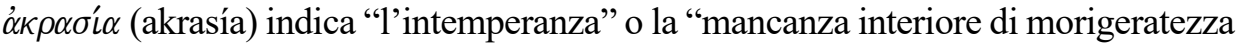
e di autocontrollo". Essa si manifesta nei desideri sessuali, ma anche nello smodato desiderio di cibo. Cioè i bicchieri e i piatti sono impuri perché contengono i beni rubati ai bisognosi; impuri sono i loro possessori che non sono capaci di controllarsi. È questa, spiegando la prima parte del v. 25 , la vera impurità dell'"interno"34.

La fine del v. 25 contiene un cambiamento inaspettato e permette una comprensione nuova della purità; questa riceve un significato etico: un atteggiamento disumano fa l'uomo più impuro.

Nella Bibbia (per es. Prov. 30,12-14), in ambito giudaico, è ampia la polemica contro coloro che stimano la purità rituale, ma trascurano la purità morale. Si ricordi in Mt 5,8 la beatitudine di coloro che sono puri di cuore e ai quali è promesso che non saranno separati da Dio; poi, in Mt 15,11.18-20, l'impurità viene "da dentro", cioè consiste nei pensieri e nelle azioni cattive che vengono dal cuore dell'uomo. Per gli ascoltatori del Vangelo, l'idea che la purità non dipenda dalle stoviglie, ma dal cuore, è chiaramente sottintesa, anche se ciò non è esplicitato. Il v. 25, all'inizio, parla dell'impurità dei piatti, ma, poi, emerge l'idea dell'impurità sociale e d'etica ${ }^{35}$.

32 Cfr. G. Michelini, Matteo. Introduzione, traduzione e commento, Cinisello Balsamo 2013, pp. 370-371.

33 Cfr. U. Luz, op. cit., pp. 422-423.

34 Cfr. Ibidem, pp. 423-424.

35 Cfr. Ibidem, pp. 423-424. 
Nel v. 25, Gesù descrive i farisei (e degli scribi) come "ipocriti". La parola

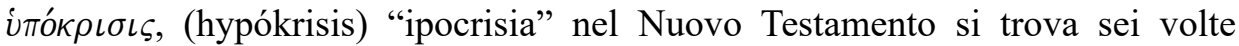
(Mt 23,28; Mc 12,15; Lc 12,1; Ga 2,13; $1 \mathrm{Tm} \mathrm{4,2;1} \mathrm{Pt} \mathrm{2,1).} \mathrm{La} \mathrm{parola} \mathrm{iток \rho \iota \tau и́}$ (hypokritếs), "ipocrita", si trova diciotto volte (Mt 6,2.5.16; 7,5; 15,7; 22,18; 23,13.14.15.23.25.27.29; 24,51; Mc 7,6; Lc 6,42; 12,56; 13,15). Quindici riferimenti alle due parole, "ipocrisia" ed "ipocrita" si trovano in Matteo; otto si trovano proprio nel capitolo $23^{36}$.

In Mt 23, otto volte ricorre la parola "ipocrita" che significa "attore" con la sfumatura di simulatore, di individuo falso ${ }^{37}$. Nel greco classico, questo termine si riferisce ad una persona che gioca, parla, recita sulla scena. L'attore, spesso, usava una maschera per esprimere i sentimenti di tristezza, gioia, rabbia. La parola "attore" assume talora un significato negativo. Gesù, usando questo termine, critica la falsità delle persone mascherate, che non sono sincere. Secondo Gesù, "ipocrita" non significa semplicemente "miscredente" o "empio", ma indica colui che vive nella contraddizione fra ciò che dice e che ciò veramente fa ${ }^{38}$.

Luz suggerisce che i farisei sono descritti come "ipocriti" perché l'accusa di "ipocrisia" è un topos classico della polemica di Matteo; inoltre, sembra che nel giudaismo i farisei siano stati accusati diffusamente di ipocrisia ${ }^{39}$.

L'invettiva del v. 26 rappresenta la possibilità per il fariseo di purificare "l'interno del bicchiere". Diventa chiaro che si dice qui del cuore e delle opere dell'uomo. Quando un uomo va d'accordo con se stesso e con il suo prossimo, raggiungerà la giusta purità del cuore e dell'agire. La parola "prima", dimostra che l'Evangelista fissa una chiara necessità e priorità, ma non è affatto capace di non considerare la purità rituale. Il versetto non descrive semplicemente le caratteristiche dei farisei e degli scribi, ma mostra anche quello che fanno nella loro vita, come agiscono. Puliscono la parte esterna della coppa, lasciando quella interna sporca. Ciò indica che Gesù, con l'immagine dei vasi impuri, vuole parlare di un'altra realtà ${ }^{40}$. Neusner sostiene che le parole di Gesù "pulisci prima l'interno del bicchiere" (Mt 23,26) si riferiscono alla legge rabbinica di purità che era scritta nella Mishnah. Secondo la Mishnah, infatti, i rabbini consideravano la parte interna della coppa più importante dalla parte esterna. La sporcizia interna della coppa causava anche l'impurità esterna, mentre la parte esterna non sporcava quella interna. Gesù è d'accordo con i rabbini: la parte interna è più

36 Cfr. Concordance to the Novum Testamentum Graece of Nestle-Aland, 26th edition, and to the Greek New Testament, 3th edition, ed. H. Bachmann, W. Slaby, Berlin-New York ${ }^{3} 1987$, p. 1847.

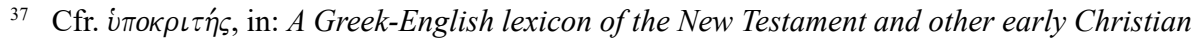
literature, op. cit., p. 1038.

38 Cfr. U. Luz, op. cit., p. 404.

39 Cfr. Ibidem, p. 405.

40 Cfr. Ibidem, pp. 424-425. 
importante della parte esterna. Tuttavia sorge la domanda: se Gesù è d'accordo con i farisei, perché litiga con loro? Secondo Neusner, i farisei che vivevano al tempo di Gesù avevano una considerazione della purezza della parte interna e di quella esterna della coppa diversa dai farisei (rabbini) che hanno scritto la Mishnah. Probabilmente, i farisei che vivevano nella prima metà del I secolo, evidenziavano l'impurità come mancanza della purezza della parte esterna dei vasi $^{41}$.

La parte interna dei vasi è sempre più importante. Questa considerazione sottolinea che bisogna prestare attenzione anche alla qualità del contenuto dei piatti. Se il piatto è riempito con del cibo acquistato ingiustamente, la purificazione non avrà valore. Il problema non sta nella purificazione dei piatti, ma nel modo in cui sono stati riempiti.

Gesù discute con i farisei non sull'importanza della purezza rituale, la purezza della parte interna o esterna, ma principalmente sul fenomeno dell'ipocrisia che può essere definito come la differenza tra ciò che è all'esterno e ciò che è all'interno dell'uomo. I rabbini insegnavano che uomo, all'interno, dovrebbe essere come all'esterno. La critica dell'ipocrisia era un tema del quale si erano occupati tante volte ${ }^{42}$.

Gesù critica la bellezza esterna delle persone che sono corrotte internamente. Anche quando parla dei sepolcri imbiancati, non è in questione l'abitudine di dipingere le tombe, ma il tema dell'ipocrisia. Secondo le metafore usate da Gesù, i suoi ascoltatori non sono invitati a riformare la legge rituale, ma a cambiare il loro comportamento, tramite il pentimento ${ }^{43}$.

Gesù si serve del motivo dei vasi per concentrarsi sulla vita morale. I vasi "pieni di rapina e di immondizia" sono un'immagine delle azioni umane ${ }^{44}$. In Mt 23,25, si parla sempre dei piatti in senso proprio. Essi sono "pieni di rapina e di immondizia" e vengono purificati. Al v. 26, Matteo parla della pulizia concreta e, poi, trasmette un significato più profondo ${ }^{45}$.

Dunque, da dove vengono i problemi fra Gesù e i farisei? Dopo l'anno 70, il Giudaismo diventò più "normativo". Era anche abbastanza naturale che i gruppi "esterni", come per esempio i cristiani, fossero in polemica con i farisei: era un modo per capire la loro propria identità. Si può pensare che i farisei rifiutassero chi seguiva Gesù. I cristiani erano considerati come un gruppo di peccatori ${ }^{46}$.

${ }^{41}$ Cfr. H. Maccoby, op. cit., p. 152.

42 Cfr. Ibidem, p. 152.

43 Cfr. Ibidem, pp. 152-153.

44 Cfr. G. A. Camp, Woe to You, Hypocrites. Law and Leaders in The Gospel of Matthew, Sheffield 2002, https://core.ac.uk/download/pdf/9848337.pdf [accesso 28.02.2018], pp. 230-231.

45 Cfr. U. Luz, op. cit., p. 421.

${ }^{46}$ Cfr. R. A. Wild, op. cit., p. 123. 
È possibile che Gesù stesso abbia creato la "tradizione farisaica". Se fosse così, tutti i dibattiti fra Gesù e $i$ farisei potrebbero essere facilmente spiegati. Secondo tale ipotesi, Gesù sarebbe considerato come un critico delle prospettive religiose dei farisei. Quando il cristianesimo diventò più stabile quale gruppo religioso che combatteva i discendenti dei farisei, la tradizione cristiana cominciò a sottolineare le differenze tra Gesù e i farisei, tacendo i punti d'accordo ${ }^{47}$.

I "guai" sono rivolti agli avversari di Gesù, ma il contesto del frammento suggerisce che essi sono pronunciati per il bene dei discepoli e sono degli insegnamenti per la comunità. Mt 23 ci permette di capire che la comunità dei cristiani ha tante preoccupazioni e che ha bisogno di indicazioni specifiche distinte dal Giudaismo ${ }^{48}$. La comunità di Matteo cerca la sua identità in autonomia dalla sinagoga: Gesù richiama all'osservanza della Torah, ma afferma che assieme ai precetti minimi, non bisogna trascurare quelli più "gravi" della legge, come: la misericordia, la fede e il giudizio. Il precetto di purità non è cancellato, ma riceve un altro valore. I "guai" sono contro la totale mancanza di comprensione per il senso delle prescrizioni rituali e delle norme che riguardano la purità ${ }^{49}$.

L'uomo contemporaneo, quando sente la parola "fariseo", la associa direttamente con il termine "ipocrita". Il fariseo è diventato sinonimo dell'ipocrisia e di un falso atteggiamento verso gli altri. Tale immagine viene spesso descritta dai Vangeli che mostrano soltanto una parte della vita dei farisei, sottolineando gli elementi negativi. I Vangeli parlano degli errori e dei peccati dei farisei, concentrandosi relativamente poco sugli elementi positivi, degni di merito.

\section{Conclusione}

Nel presente lavoro, riconducibile a Mt 23,25-26, si è cercato di dimostrare che Gesù non critica i farisei che rispettano le regole della purezza ma, critica che la formalizzata purezza rituale abbia preso il posto della moralità, che esige lo sforzo di cambiamento spirituale dell'uomo. Questo è concorde con la tradizione giudaica che dà un valore etico alla purità rituale, cioè l'uomo diventa più impuro quando non osserva la Legge, se viene meno la purità rituale. Gesù indica, piuttosto, che cosa è peccato nell'atteggiamento dell'uomo, non solo nella vita di un fariseo. I "guai" creano una invettiva nel senso di un severo rimprovero. I "guai" detti da Gesù sono rivolti a chi lo osteggia, ma il contesto ci permette capire

47 Cfr. Ibidem, p. 124.

48 Cfr. D. E. Cook, A Gospel Portrait of the Pharisees, "Review \& Expositor" 84/2(1987), pp. 230-231.

49 Cfr. F. De Carlo, Vangelo secondo Matteo. Nuova versione, introduzione e commento, Cinisello Balsamo 2016, p. 47. 
che essi sono pronunziati per il bene dei discepoli e sono degli insegnamenti per la comunità. Lui rimprovera perché vuole la conversione dell'uomo. Ognuno dovrebbe pentirsi, cambiare il suo comportamento, abbandonare il sentiero dell'ingiustizia, dell'ipocrisia e vivere secondo il Vangelo annunciato dal Figlio di Dio. Purificare "l'interno del bicchiere" significa osservare sinceramente la Legge, ingaggiare pienamente se stesso nella realizzazione della volontà di Dio. Soltanto così la vita dell'uomo trova il suo significato vero e puro ${ }^{50}$. Gesù presenta l'uso metaforico delle immagini della coppa e del piatto in riferimento alla vita dell'uomo: come tali cose di uso quotidiano sono lavate prima nella parte interna e poi in quella esterna, così anche l'uomo deve realizzare un cammino di purificazione nella propria anima prima di preoccuparsi di essere perfetto agli occhi degli altri ${ }^{51}$. Mt 23,25-26 riguarda la purezza vera, quella del cuore e di ogni attività dell'uomo.

\section{Czego dotyczy krytyka faryzeuszy w Mt 23,25-26?}

\section{Streszczenie}

W Nowym Testamencie faryzeusze ukazywani są zazwyczaj jako wrogowie i nieprzyjaciele Jezusa. Ich prezentacja jest raczej tendencyjna i jednostronna, ograniczona do kwestii kontrowersyjnych. Ewangelie zazwyczaj nie ukazują tego, co było w ich życiu pozytywnego i zgodnego z życiem chrześcijanina. Jezus we fragmencie Mt 23,25-26 nie tyle krytykuje faryzeuszów za to, że respektują reguły czystości rytualnej w życiu codziennym, lecz ukazuje, czym jest grzech w życiu człowieka, nie tylko w życiu faryzeusza, ale każdego. Każdy jest wezwany do tego, aby się nawrócić, zmienić swoją postawę, odrzucić hipokryzję i żyć według zasad Ewangelii. Jezus używa metafory kubka i misy, aby w prosty sposób ukazać swoim słuchaczom, że tak jak w przypadku przedmiotów, których używa się w codzienności, najpierw myje się ich stronę wewnętrzną, a potem zewnętrzną, tak samo w przypadku człowieka, powinien on najpierw oczyścić swoją duszę, a potem pokazać się dobrym wobec innych.

\section{Slowa kluczowe}

Jezus, czystość rytualna, faryzeusze, konflikt, moralność, nawrócenie

50 Cfr. J. Homerski, Ewangelia wedtug św. Mateusza. Wstęp - przekład z oryginału. Komentarz, Poznań 2004, p. 307.

${ }_{51}$ Cfr. A. S. Romano, op. cit., p. 198. 


\section{What is the criticism of the Pharisees in Mt 23:25-26?}

\section{Summary}

In the New Testament, the Pharisees are usually presented as enemies and foes of Jesus. Their presentation is rather biased and one-sided, limited to controversial issues. The Gospels usually fail to show what positive in their lives and in harmony with that of a Christian was. In the passage of Mt 23:25-26, Jesus does not so much criticize the Pharisees for respecting the rules of ritual purity in their daily lives, but shows what $\sin$ in human life is, not only in the life of a Pharisee, but everyone. Everyone is called to convert, change its attitude, reject hypocrisy and live according to the Gospel principles. Jesus uses the metaphor of a cup and a dish to easily show his listeners that, as in the case of everyday objects, which first they are washed inside, then outside, exactly the same should happen with a man, he should first purify his soul and then show himself good towards others.

\section{Keywords}

Jesus, ritual purity, Pharisees, conflict, hypocrisy, morality, conversion

\section{Bibliography}

A Greek-English lexicon of the New Testament and other early Christian literature, ed. W. Bauer, F. W. Danker, W. F. Arndt, F. W. Gingrich, Chicago ${ }^{3} 2000$.

Bosak P. C., Leksykon wszystkich postaci biblijnych, Kraków 2015.

Bultmann R., The History of the Synoptic Tradition, New York ${ }^{2} 1968$.

Camp G. A., Woe to You, Hypocrites. Law and Leaders in The Gospel of Matthew, Sheffield 2002, https://core.ac.uk/download/pdf/9848337.pdf [accesso 28.02.2018].

Concordance to the Novum Testamentum Graece of Nestle-Aland, 26th edition, and to the Greek New Testament, 3th edition, ed. H. Bachmann, W. Slaby, Berlin-New York ${ }^{3} 1987$.

Cook D. E., A Gospel Portrait of the Pharisees, "Review \& Expositor" 84/2(1987), pp. 221-233.

Dąbrowski E., Nowy Testament na tle epoki, t. 1, Poznań ${ }^{2} 1965$.

De Carlo F., Vangelo secondo Matteo. Nuova versione, introduzione e commento, Cinisello Balsamo 2016.

Dictionary of Jesus and the Gospels, ed. J. B. Green, J. K. Brown, N. Perrin, Downers Grove 1992.

Green W. S., What do we really know about the Pharisees, and how do we know it?, in: Quest of the Historical Pharisees, ed. J. Neusner, B. D. Chilton, Waco 2007, pp. 409-423 .

Harrington H. K., Did the Pharisees eat ordinary food in a state of ritual purity, "Journal for the Study of Judaism" 26/1(1995), pp. 42-54. 
Homerski J., Ewangelia wedtug św. Mateusza. Wstęp - przekład z oryginatu. Komentarz, Poznań 2004.

Josephus, Jewish Antiquities, Vol. VII: Books 16-17, trans. R. Marcus, A. Wikgren. Loeb Classical Library 410. Cambridge (MA), 1963.

Kern K., We are the Pharisees, Scottdale 1995.

Luz U., Vangelo di Matteo, Vol. 3, Brescia 2013.

Maccoby H., Ritual and morality. The Ritual Purity System and its Place in Judaism, Cambridge 1999.

Michelini G., Matteo. Introduzione, traduzione e commento, Cinisello Balsamo 2013.

Neusner J., From Politics to Piety: The Emergence of Pharisaic Judaism, New York 1973.

Newport K. G., The Pharisees In Judaism prior to $a$. $d$. 70, "Andrews University Seminary Studies" 29/2(1991), pp. 127-137.

Paciorek A., Ewangelia wedlug św. Mateusza. Rozdzialy 14-28. Wstęp, przekład z oryginatu, komentarz, t. 2, Częstochowa 2008.

Polak P., Fundamentalizm faryzejski a radykalizm ewangeliczny, "Eryda" 1(1)2015, pp. 44-60.

Rakocy W., Faryzeusze. Historia - Ewangelie, Lublin 2002.

Rakocy W., Obraz i funkcja faryzeuszy w dziele Lukaszowym ( $₫ k-D z)$. Studium literacko-teologiczne, Lublin 2000.

Reymond P., Dizionario di ebraico e aramaico biblico, Roma ${ }^{2} 2012$.

Romano A. S., Purità ed impurità tra ebraismo e tradizioni sinottiche. Casi di impurità corporale, https://www.academia.edu/31802505/Purit\%C3\%A0_e_impurit\%C3\%A0 tra_ebraismo_e_tradizioni_sinottiche_Casi_di_impurit\%C3\%A0_corporale [accesso 05.01.2021].

Rosik M., Śladami faryzeuszy i saduceuszy, "Ziemia Święta” 2(2007)50, pp. 16-19.

Rubinkiewicz R., Wprowadzenie do apokryfów starego Testamentu, Lublin 1987.

Saldarini A. J., Farisei, scribi e sadducei nella società palestinese, Brescia 2003.

Sievers J., Chi era un fariseo? Un approccio prosopografico, "Poznańskie Studia Teologiczne" 27(2013), pp. 13-27.

Wild R. A., The encounter between Pharisaic and Christian Judaism: some early Gospel evidence, "Novum Testamentum" 27/2(1985), pp. 105-124. 